\title{
FEASIBILITY OF INCREASING THE ENERGY OF RHIC*
}

\author{
W.W. MacKay ${ }^{\dagger}$, A. Jain, A.U. Luccio, F. Pilat, T. Roser, \\ S. Tepikian, D. Trbojevic, BNL, USA
}

\section{Abstract}

In this paper we discuss the possibility of increasing the energy of beams in RHIC by as much as $30 \%$ with a modest trade-off in luminosity. The arc dipoles and quadrupoles were designed with considerable margin. For higher energies $\left(>100 \mathrm{GeV} /\right.$ nucleon) the minimum $\beta^{*}$ may be required to increase as the interaction region triplets saturate. The separator magnets (DX) have the least margin for increased field, so we consider three scenarios: allowing for a small crossing angle with the present DX magnets, upgrading the DX magnets to higher strength, and permitting a crossing angle of $\sim 1^{\circ}$ by removing the DX magnets altogether.

\section{INTRODUCTION}

The Relativistic Heavy Ion Collider (RHIC)[1] was designed to collide ion species from protons up to gold ions. For the design the maximum rigidity per beam is $p / q=$ $839.5 \mathrm{~T} \cdot \mathrm{m}$ which corresponds to about $100 \mathrm{GeV} /$ nucleon for gold ions or $250 \mathrm{GeV} / \mathrm{c}$ for protons. The main bending magnets in the arcs were built with a considerable margin of $30 \%$ or more in field capability.

Since the two beams are in separate rings (except for the common separator magnets) it is possible to collide different species, provided that energies and orbits are adjusted to keep the collisions synchronous at the interaction point. For proton-ion collisions, the separator magnets (DX) must be shifted horizontally. In this paper we consider collisions of equal species, although unequal species are not ruled out.

The interaction region (IR) layout for one ring is shown schematically in Fig 1 with design geometrical parameters listed in Table 1. The focusing triplets are on either side of the IR outside of these four bends.

\begin{tabular}{|cc|}
\hline$t$ & $0.238 \mathrm{~m}$ \\
$a$ & $7.007 \mathrm{~m}$ \\
$b$ & $19.600 \mathrm{~m}$ \\
$l_{x}$ & $3.700 \mathrm{~m}$ \\
$l_{0}$ & $3.589 \mathrm{~m}$ \\
$\theta_{x}$ & $18.861 \mathrm{mrad}$ \\
$\theta_{0}$ & $15.186 \mathrm{mrad}$ \\
\hline
\end{tabular}

Table 1: Parameters for the IR as shown in Fig. 1

In the design, the two beams intersect colinearly in a $19.6 \mathrm{~m}$ long straight section through the IR as shown in Fig. 2.

\footnotetext{
* Work supported in part by the U.S. Department of Energy.

†waldo@bnl.gov
}

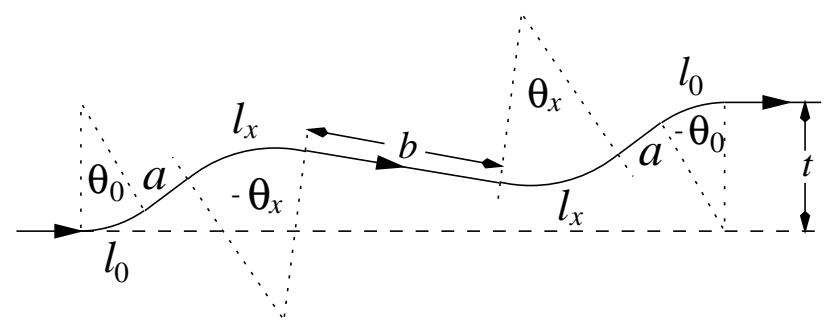

Figure 1: Design trajectory for a single beam through the D0 and DX magnets.

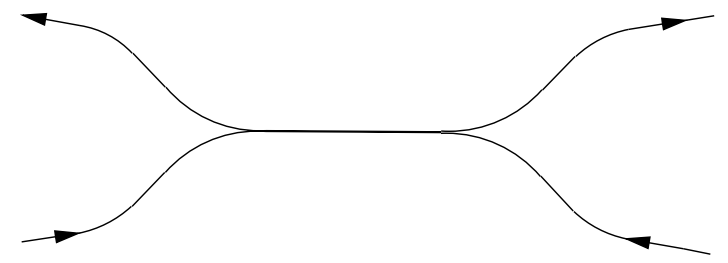

Figure 2: Schematic of trajectories through the IR with collinear beams.

\section{NO CROSSING ANGLE}

The nominal magnetic fields for the design and estimated maximum quench fields for the bends are presented in Table 2. In RHIC, the bend magnets have three different cross sections: and arc dipole with a coil i.d. of $8 \mathrm{~cm}$, a D0 magnet with a $10 \mathrm{~cm}$ i.d., and a DX magnet with an $18 \mathrm{~cm}$ i.d. If we consider a $0^{\circ}$ crossing angle with the present con-

\begin{tabular}{|c|l|l|c|}
\hline Dipole & $B_{\text {nom }}[\mathrm{T}]$ & $B_{\mathrm{q}}[\mathrm{T}]$ & $B_{\mathrm{q}} / B_{\text {nom }}$ \\
\hline Arc dipole & $3.46 \mathrm{~T}$ & $4.6 \mathrm{~T}$ & 1.33 \\
D0 dipole & 3.55 & 4.42 & 1.24 \\
DX dipole & 4.28 & 5.2 & 1.22 \\
\hline
\end{tabular}

Table 2: Design fields $B_{\text {nom }}$ and estimated quench fields $B_{\mathrm{q}}$ of the bend magnets.[1]

figuration of magnets, but with power supplies upgraded to allow maximum fields, then the maximum energy would be limited by the DX magnets with the D0 magnets close behind. In this case we might be able to increase the collision energy by just over $20 \%$ without replacing the DX and D0 magnets.

\section{CROSSING ANGLE}

By allowing a little crossing angle at the interaction point the bend angles $\theta_{x}$ and $\theta_{0}$ may be reduced, thus increasing the maximum achievable energy. For a given beam the design trajectories through the incoming and outgoing triplets 


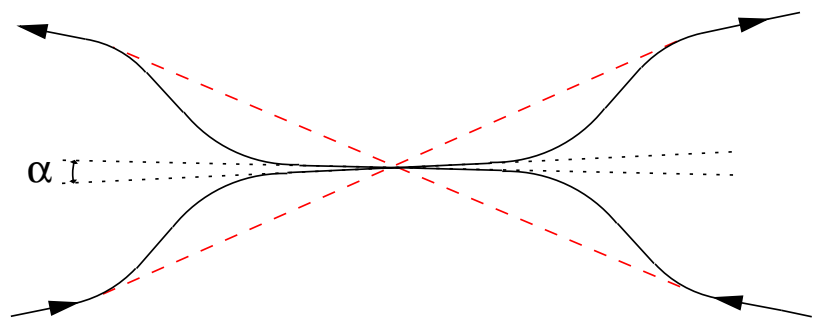

Figure 3: Trajectories through the IR with a crossing angle $\alpha$. The dashed (red) lines show how the crossing angle increases without the DX magnets.

are parallel so the bends through the pairs of D0 and DX magnets must cancel:

$$
\theta_{0}-\theta_{x}+\theta_{x}-\theta_{0}=0
$$

as shown in Fig1. With the double-S bends through the IR, the design trajectory of the outgoing beam is transversely displaced by an amount

$t=2 l_{0} \sin \frac{\theta_{0}}{2}+2 a \sin \theta_{0}+b \sin \left(\theta_{0}-\theta_{x}\right)+2 l_{x} \sin \left(\theta_{0}-\frac{\theta_{x}}{2}\right)$.

For small angles this may be linearized to give

$$
\theta_{0} \simeq \frac{t+\left(b+l_{x}\right) \theta_{x}}{2\left(a+l_{x}\right)+b+l_{0}} .
$$

In the design case the other beam has a similar but flipped and rotated trajectory so that the beams are collinear between the DX magnets as shown in Fig. 2.

A crossing angle $\alpha$ as shown in Fig. 3 is given by

$$
\alpha=2\left(\Delta \theta_{x}-\Delta \theta_{0}\right),
$$

where $\Delta \theta_{x}$ and $\Delta \theta_{0}$ are deviations from the design bend angles of the DX and D0 magnets. If we require that the orbit around the rest of the machine remains unchanged, then

$$
\frac{d \alpha}{d \Delta \theta_{x}}=-2 \frac{l_{0}+l_{x}+2 a}{2\left(a+l_{x}\right)+b+l_{0}} \simeq-0.955 .
$$

The maximum crossing angle is limited by the aperture of the beam tube at the end of the DX magnet as shown in Fig. 4. Using a small angle approximation the clearance of the beam at closest approach to the aperture at the end of the DX magnet is approximately

$$
k \approx r-\left(l_{x}+\frac{b}{2}\right) \frac{\alpha}{2}-\frac{l_{x} \theta_{x}}{2}-g\left(\frac{\alpha}{2}+\theta_{x}\right),
$$

where $r=6.985 \mathrm{~cm}$ is the inner radius of the DX beam pipe which extends $g=25.6 \mathrm{~cm}$ beyond the end of the magnet before enlarging. For a $130 \mathrm{GeV} /$ nucleon gold beam with an emittance of $\pi \epsilon_{95 \%}^{N}=40 \pi \mu \mathrm{m}$ with $\beta^{*}=1 \mathrm{~m}$ at the interaction point, the rms beam size at the end of the DX magnet is $3.0 \mathrm{~mm}$. To have at least $\pm 6 \sigma$ of clearance for the beam the maximum allowed crossing angle is then $2.5 \mathrm{mrad}$. The fields for the D0 and DX magnets would be $B_{\mathrm{D} 0} \simeq 4.37 \mathrm{~T}$ and $B_{\mathrm{DX}} \simeq 5.12 \mathrm{~T}$. The quench fields from Table 2 give a lower limit to the crossing angle of $1.3 \mathrm{mrad}$.

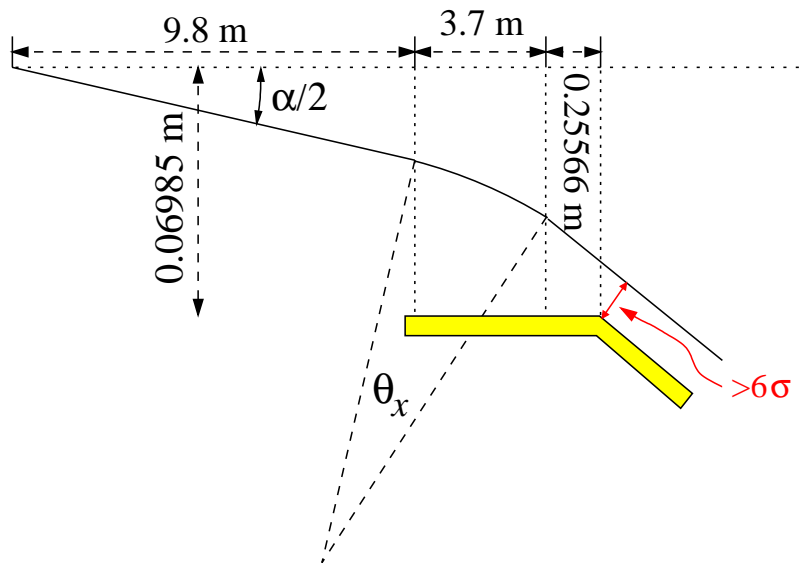

Figure 4: Aperture limit of trajectory through DX magnet. The warm beam pipe extends about $25.6 \mathrm{~cm}$ beyond the end of the DX magnet before bending towards the D0 magnet.[2]

\subsection{Without DX magnets}

Another solution would be to remove the DX magnet altogether, and realign the D0 magnets for an optimized aperture with a crossing angle of $\alpha=18 \mathrm{mrad}$ as shown by the dashed lines of Fig. 3. In this case we only need $\theta_{0}=5.33 \mathrm{mrad}$, so the limiting magnets are just the arc dipoles.

\section{OTHER MAGNETS}

The quadrupoles have a quench to design field ratios of around 50\%, and should be able to reach energies $30 \%$ higher than design.

At the $30 \%$ increased energy, the arc dipoles would operate at $7020 \mathrm{~A}$ and have a measured sextupole component[3] of $b_{2}=-0.64 \times 10^{-4} \mathrm{~cm}^{-2}$. The sextupole magnets for RHIC were overdesigned. In order to correct total chromaticities at $130 \mathrm{GeV} /$ nucleon the sextupole magnets need to operate at only $20 \%$ of their maximum current. Removing the DX magnets has almost no effect on chromaticity.

For polarized protons[4], the helical Siberian snakes[5] are essentially independent of energy with only a small dependence of the spin rotation on $v / c$. Since the IR straight section is not parallel to the straight section containing the spin rotator and triplet, there is a dependence on energy. To achieve longitudinal polarization at the interaction point, the rotator must rotate the polarization from vertical into the horizontal plane at an angle $\Psi=\frac{g-2}{2} \gamma\left(\theta_{x}-\theta_{0}\right)$ relative to the beam's direction allowing the D0 and DX magnets to finish the precession into the longitudinal direction. Table 4 lists the required values of $\Psi$ for the three cases with polarized protons at $325 \mathrm{GeV}$. A preliminary check indicates that the rotators should be able to produce longitudinal polarization at the STAR and PHENIX detectors with all three scenarios, although for the 18 mrad crossing angle the rotator power supplies should be reversed. A 


\begin{tabular}{|c|c|c|c|c|}
\hline$\alpha[\mathrm{mrad}]$ & 0.0 & 1.3 & 2.5 & 18.0 \\
$\theta_{x}-\theta_{0}[\mathrm{mrad}]$ & 3.675 & 3.020 & 2.423 & -5.33 \\
$\Psi$ & $130.8^{\circ}$ & $99.0^{\circ}$ & $86.2^{\circ}$ & $-189.6^{\circ}$ \\
\hline
\end{tabular}

Table 3: Required polarization angle for a spin rotator.

more careful analysis of the effects on the polarized proton program if and when an energy upgrade is planned.

\section{CONCLUSIONS}

It may be possible to increase the center-of-mass energy of RHIC by as much as $30 \%$ by upgrading the main dipole and quadrupole power supplies for higher operating currents. Since the estimated quench field of the arc dipoles is only $33 \%$ higher than the design field, the actual increase in energy may be less without considerable training of magnets.

In order to have a $0^{\circ}$ crossing angle with the present DX and D0 magnets, the increase would probably be less than $20 \%$.
Operation with a crossing angle between 1.3 and $2.5 \mathrm{mrad}$ at $130 \mathrm{GeV} /$ nucleon could be possible with the present D0 and DX magnets. A simple solution with a $18 \mathrm{mrad}$ crossing angle is obtained by discarding the DX magnets and realigning the D0 magnets.

The other existing magnets such as sextupoles, correctors, snakes, and rotators should be able to meet the requirements of a $30 \%$ increase in energy.

\section{REFERENCES}

[1] RHIC Design Manual (http://www.agsrhichome.bnl.gov/NTshare/rhicdm/)

[2] S. Peggs et al., "Interaction Region Closed Orbits", RHIC/AP/135 (1997).

[3] Fulvia Pilat, "RHIC Magnet Transfer Functions", Technical Note, RHIC/AP/113, (1996) BNL, Upton NY.

[4] A. Alekseev et al., "Polarized Proton Collider at RHIC", to be submitted to NIM.

[5] W.W. MacKay et al., "Superconducting Helical Snake Magnets: Construction and Measurments", DESY-PROC-199903, p. 163 (1999). 